\title{
INIBIDOR DA AÇÃO DO ETILENO NA CONSERVAÇÃO PÓS-COLHEITA DE Chrysanthemum morifolium RAMAT cv. DRAGON
}

\author{
Inhibitor of ethylene action in postharvest conservation of \\ Chrysanthemum morifolium Ramat cv. Dragon \\ Poliana Cristina Spricigo ${ }^{1}$, Ben-Hur Mattiuz ${ }^{2}$, Júlia de Pietroº , \\ Claudia Fabrino Machado Mattiuz ${ }^{3}$, Maria Elisa Morais de Oliveira ${ }^{4}$
}

\begin{abstract}
RESUMO
A durabilidade e a qualidade pós-colheita de flores de corte são atributos fundamentais na sua valoração ao longo da cadeia produtiva e na satisfação dos consumidores. Objetivou-se, nesta pesquisa, verificar o efeito do tiossulfato de prata, associado ou não à sacarose, na manutenção da qualidade pós-colheita de hastes de crisântemos (Chrysanthemum morifolium Ramat cv. Dragon) . O experimento foi conduzido sob delineamento inteiramente casualizado, em esquema fatorial testando soluções de manutenção com tiossulfato de prata (STS), sob cinco níveis (Água destilada; STS a 0,2 mM; STS a 0,2 mM + sacarose a $50 \mathrm{~g} \mathrm{~L}^{-1}$; STS a $0,4 \mathrm{mM}$; STS a $0,4 \mathrm{mM}$ + sacarose a $\left.50 \mathrm{~g} \mathrm{~L}^{-1}\right)$; e data de amostragem, por três níveis $(0 ; 3 ; 6$ dias). Utilizaram-se três repetições com duas hastes florais em cada tratamento. Foram feitas avaliações físicas: coloração, massa fresca e conteúdo relativo de água (CRA); avaliações químicas: açúcares redutores e pigmentos; e avaliações qualitativas: turgescência, cor das flores, e número de botões, flores entreabertas e abertas. O tratamento com $0,2 \mathrm{mM}$ de STS possibilitou melhor manutenção da massa fresca das hastes. A concentração de pigmentos e carboidratos redutores foi maior naqueles tratamentos em que a sacarose foi associada. A coloração e o conteúdo relativo de água foram favorecidos nos tratamentos STS a 0,2 e 0,4mM. A concentração de $0,2 \mathrm{mM}$ de STS foi a que obteve os melhores resultados, prolongando a vida de vaso das hastes. A qualidade das hastes foi superior, tendo as melhores avaliações de conteúdo de água, cor e turgescência.
\end{abstract}

Termos para indexação: Flor de corte, tiossulfato de prata, sacarose.

\section{ABSTRACT}

The durability and postharvest quality of cut flowers are fundamental attributes in value along the production chain and in consumer satisfaction. The objective of this study was to evaluate the effect of chemical inhibitors of ethylene action on maintaining the postharvest quality of chrysanthemum stems (Chrysanthemum morifolium Ramat cv. Dragon). The experiment tested maintenance solutions with silver thiosulfate (STS) under five levels (distilled water, a $0.2 \mathrm{mM} \mathrm{STS}$, the STS $0.2 \mathrm{mM}+$ sucrose at $50 \mathrm{~g} \mathrm{~L}^{-1}$, STS at 0.4 $\mathrm{mM}$; STS at $0.4 \mathrm{mM}+$ sucrose at $\left.50 \mathrm{~g} \mathrm{~L}^{-1}\right)$, and date of sampling, for three levels $(0,3,6$ days). Three replications with two flower stems in each treatment were used in the experiment. Physical assessments were made: color, fresh mass and relative water content; chemical evaluations: reducing sugars and pigments, and qualitative assessments: turgidity, flower color, and number of buds, open flowers and partially open flowers. Treatment with $0.2 \mathrm{mM}$ STS resulted in better maintenance of fresh mass of stems. The concentration of pigments and reducing sugar was higher in those treatments in which sucrose was associated. The color and relative water content were favored in treatments STS $0.2 \mathrm{mM}$ and $0.4 \mathrm{mM}$. The concentration of $0.2 \mathrm{mM}$ STS obtained the best results, prolonging the vase life the stems. The quality of these stems was higher, with the best assessments of water content, color and turgidity.

Index terms: Cut flower, silver thiosulfate, sucrose.

(Recebido em 16 de junho de 2009 e aprovado em 8 de março de 2010)

\section{INTRODUÇÃO}

No Brasil, parte da produção de flores de corte é perdida após a saída do produto do local de cultivo. Flores de corte possuem alta perecibilidade e são muito suscetíveis a danos, sendo assim, o manuseio pós-colheita adequado das hastes é imprescindível e colabora para evitar perdas que são expressivas no setor da floricultura.
A perda de qualidade das flores cortadas está relacionada a vários processos fisiológicos (Halevy \& Mayak, 1974). O balanço hídrico é considerado o fator determinante do comportamento e da longevidade dos órgãos das plantas (Borochov et al., 1982), ressaltando-se que a falta de água no organismo acelera a senescência (Mayak, 1987).

\footnotetext{
UUniversidade Estadual de Campinas/UNICAMP - Faculdade de Engenharia Agrícola - Avenida Candido Rondon - 501 - Cidade Universitária Zeferino Vaz - 13083-875 - Barão Geraldo - Campinas, SP - polianaspricigo@yahoo.com.br

2Universidade Estadual Paulista/ UNESP - Jaboticabal, SP

${ }^{3}$ Centro Universitário Moura Lacerda - Ribeirão Preto, SP

${ }^{4}$ Universidade Estadual de Campinas/UNICAMP - Faculdade de Engenharia Agrícola - Campinas, SP
} 
A sacarose favorece o balanço hídrico das flores cortadas acumulando-se nas flores, aumentando a concentração de solutos osmoticamente ativos e, consequentemente favorecendo a manutenção da turgescência das pétalas (Halevy, 1976). O fornecimento de açúcar exógeno retarda a senescência das flores (Paulin, 1986), substituindo o carboidrato endógeno esgotado pela respiração (Nowak et al., 1991).

Ressalta-se ainda que, tratamentos com inibidores de etileno, são amplamente utilizados para aumentar a durabilidade de flores cortadas. O tiossulfato de prata (STS) bloqueia a elevação do teor de etileno, que normalmente ocorre antes do murchamento de pétalas de cravos. A elevação na produção de dióxido de carbono, subsequente ao aumento na produção de etileno, foi completamente suprimida após o pré-tratamento com tiossulfato de prata a 0,2 $\mu \mathrm{M}$ (Venn, 1979).

Objetivou-se, neste trabalho avaliar o efeito de produtos químicos inibidores da ação do etileno, em soluções de manutenção, associados ou não à sacarose, na manutenção da qualidade pós-colheita de hastes de crisântemos (Chrysanthemum morifolium Ramat cv. Dragon).

\section{MATERIAL E MÉTODOS}

Para a condução do experimento foram empregadas hastes florais de Chrysanthemum morifolium cv. Dragon, colhidas no ponto de colheita comercial e transportadas a seco do município de Holambra até o Laboratório de Tecnologia dos Produtos Agrícolas da FCAV-UNESP, Campus de Jaboticabal-SP. No laboratório, as flores foram submetidas à toalete eliminando-se folhas basais, e à padronização a $50 \mathrm{~cm}$ de altura mediante o corte das hastes dentro de recipientes com água destilada. Após a padronização, as flores foram etiquetadas, pesadas e submetidas aos tratamentos: 1) água destilada; 2) STS a $0,2 \mathrm{mM}$; 3) STS a $0,2 \mathrm{mM}$ + sacarose a $50 \mathrm{~g} \mathrm{~L}^{-1}$; 4) STS a 0,4 $\mathrm{mM}$; 5) STS a $0,4 \mathrm{mM}+$ sacarose a $50 \mathrm{~g} \mathrm{~L}^{-1}$.

$\mathrm{O}$ experimento foi conduzido em delineamento experimental inteiramente casualizado, num esquema fatorial composto por dois fatores: cinco tratamentos e três datas de avaliação $(0 ; 3 ; 6$ dias). Foram utilizadas três repetições com duas hastes florais cada uma, perfazendo um total de seis hastes por tratamento.

A avaliação da qualidade das inflorescências foi realizada periodicamente através de atribuição de notas (observação visual) às folhas e flores senescentes, conforme o critério a seguir. Turgescência: nota $4=$ flores e folhas túrgidas; nota 3 = levemente murcha (murchamento em até $25 \%$ das flores e folhas); nota $2=$ murcha (murchamento em 25-50\% das flores e folhas); nota $1=$ muito murcha (murchamento em mais de $50 \%$ das flores e folhas). Cor das flores: nota 4 = cor viva; nota $3=$ levemente desbotada; nota 2 = desbotada; nota 1 = muito desbotada. Número de botões, flores entreabertas e abertas: foram contadas as quantidades de botões, flores entreabertas e abertas a cada avaliação. A vida de vaso foi considerada encerrada quando $50 \%$ ou mais flores de uma haste/ repetição apresentaram-se sem atrativos comerciais.

Em relação aos aspectos físicos, a coloração foi determinada usando-se o reflectômetro Minolta CR 200b, conforme o sistema da CIE 1976 (Minolta, 1994), o que permitiu calcular a luminosidade (L), o ângulo de cor $\left(\mathrm{h}^{\circ}\right) \mathrm{e}$ a Cromaticidade (C). Para avaliação da massa fresca, as flores foram pesadas em balança analítica. O conteúdo relativo de água (CRA) foi determinado de acordo com a metodologia descrita por Kramer (1983). Nos crisântemos em estudo foram retiradas 10 pétalas (lígulas) de cada flor, em cada tratamento. Em seguida, as pétalas foram pesadas e imersas em água destilada, para hidratação por um período de 4 horas. Após esse período, as pétalas foram colocadas em pesa-filtro com capacidade para $100 \mathrm{~mL}$, pesados e levados para estufa com circulação de ar forçada, a $70^{\circ} \mathrm{C}$, para secagem, o que permitiu o cálculo do conteúdo relativo de água, expresso em porcentagem.

As avaliações químicas consistiram na determinação de açúcares redutores, conforme metodologia de Honda et al. (1982) e Chantrachit (1999). Além dos carboidratos, determinaram-se os pigmentos, na qual foram retiradas amostras das partes florais (lígulas) do crisântemo, pesando $1 \mathrm{~g}$, para determinação do conteúdo de antocianina, segundo a metodologia descrita por Francis (1982), expressa em mg $100 \mathrm{~g}^{-1}$.

\section{RESULTADOS E DISCUSSÃO}

Conforme a Figura 1, nota-se que a perda de massa fresca das hastes de crisântemos variou entre os tratamentos testados. Essa variação foi menor para o tratamento STS 0,2 mM, entretanto, quando associado à sacarose verifica-se o efeito contrário, apresentando a maior perda de massa fresca entre os tratamentos. Todos os tratamentos apresentaram grande variação acumulada na massa fresca, ou seja, nenhum efetivamente colaborou para a manutenção da massa fresca.

Para Barbosa et al. (2005), a aplicação de STS não influenciou o número médio de dias para a vida de prateleira da variedade Rage, de minicrisântemos, também, a concentração de $0,5 \mathrm{mM}$ de STS foi insuficiente para aumentar o período da vida de vaso das variedades Summer Time e Davis. 


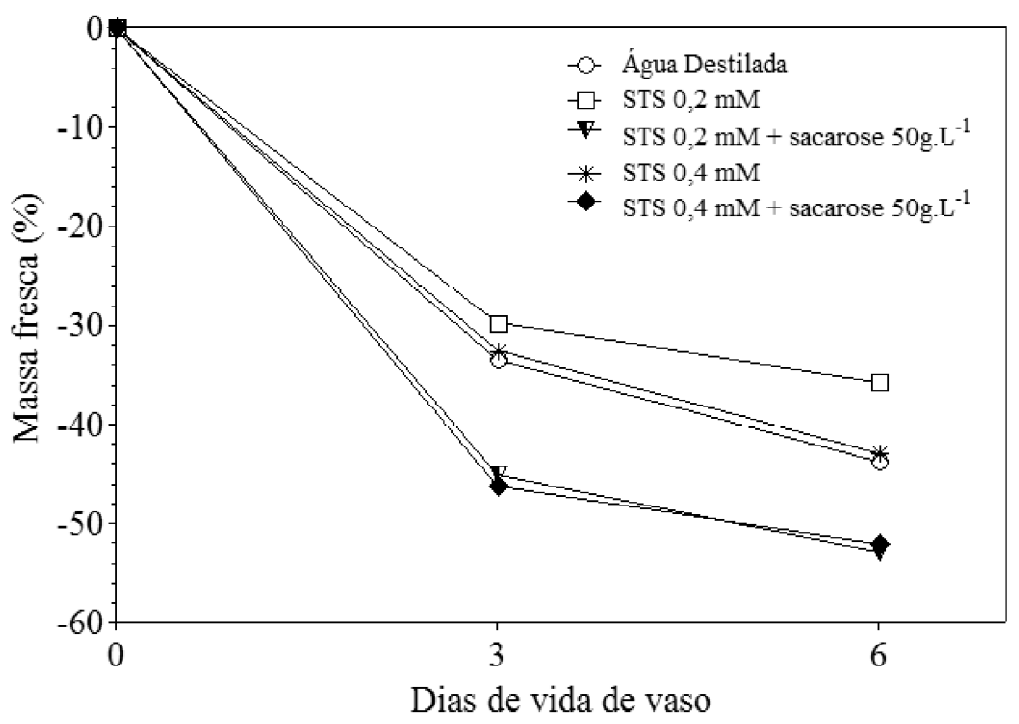

Figura 1 - Variação acumulada na massa fresca em lígulas de crisântemos, submetidas a cinco soluções de manutenção e armazenadas por 12 dias a $22^{\circ} \mathrm{C}$.

Hastes de crisântemos pré-tratadas com STS + 10\% de sacarose inibiram a síntese de etileno e proporcionaram qualidade pós-colheita (Burzo \& Dobrescu, 1995). Hassan \& Schmidt (2005), testando soluções que continham STS a $0,4 \mathrm{mM}$ adicionada de sacarose, obteve melhor manutenção na massa fresca de hastes de crisântemos, com diferença de $14,45 \%$ sobre o tratamento controle. Inflorescências de copode-leite, submetidas à refrigeração, obtiveram desempenho semelhantes quando mantidas em água ou em conservantes comerciais, apresentando características desejáveis de qualidade até o $11^{\circ}$ de armazenamento (Almeida et al., 2008).

Para as hastes de crisântemos avaliadas neste experimento, as maiores perdas de massa fresca foram proporcionadas pelas soluções que associavam STS e sacarose. Isso pode ter ocorrido por possível sensibilidade da cultivar Dragon aos compostos dessa combinação, possível bloqueio no sistema vascular ou ainda favorecimento do desenvolvimento de microrganismos que possam ter influenciado na absorção de água pela haste.

Pelos dados apresentados na Tabela 1 , verifica-se que as soluções de manutenção afetaram os teores de antocianinas nas lígulas das hastes de crisântemos, diferindo entre si e ao longo do armazenamento. Dentre os tratamentos, as soluções associadas à sacarose foram as que apresentaram as maiores médias. Isso está relacionado a maior perda de massa fresca das hastes ocorridas ao longo do período, fazendo com que uma quantidade maior de material fosse amostrada para efetuar as análises, com consequente aumento nos valores de antocianina, verificado pela evolução das médias, ao longo do armazenamento. A diferença nos níveis de antocianinas dos tratamentos com sacarose podem estar também associadas a alguma interação entre o STS e a sacarose, favorecendo o acúmulo de antocianinas.

Ainda na Tabela 1, é possível observar que as médias para os carboidratos redutores diferiram entre os tratamentos, e essas se apresentaram maiores naquelas soluções que tiveram associação de sacarose. O conteúdo de glicose em pétalas de crisântemos do tratamento controle foi de $1,87 \mathrm{mg} \mathrm{g}^{-1}$, enquanto que, em tratamentos com STS, esse valor era de 4,12 $\mathrm{mg} \mathrm{g}^{-1}$ aos 7 dias, conforme relatado por Hassan \& Schmidt (2005). Em alguns casos, a sacarose pode causar efeitos adversos. Moraes et al. (1999) observaram que a sacarose de 1 a $2 \%$ na solução de vaso é responsável pela plasmólise e morte do tecido de folhas de rosas de corte. Liao et al. (2000) constataram que a utilização do STS associado à sacarose não apresentou resultados tão satisfatórios quanto à associação de 8-HQS e sacarose, para rosas. Para esses autores, STS + sacarose não foram suficientes para inibir a ação do etileno na senescência das rosas, porém obtiveram resultados superiores aos da água destilada.

Pelos dados de CRA das hastes (Tabela 1), notase que houve diferenças significativas entre os tratamentos, onde a solução de STS a $0,2 \mathrm{mM}$ foi a que mais manteve a turgidez, diferindo significativamente da testemunha que obteve as menores médias para o parâmetro avaliado. 
Tabela 1 - Teor de antocianinas, carboidratos redutores e conteúdo relativo de água (CRA) de hastes de crisântemos submetidas a cinco soluções de manutenção e armazenadas por 12 dias a $22^{\circ} \mathrm{C}$.

\begin{tabular}{|c|c|c|c|}
\hline \multirow{2}{*}{ Variável } & \multirow{2}{*}{$\begin{array}{l}\text { Antocianina } \\
\mathrm{mg} 100 \mathrm{~g}^{-1}\end{array}$} & Carboidratos redutores & \multirow{2}{*}{$\begin{array}{l}\text { CRA } \\
(\%)\end{array}$} \\
\hline & & $\mathrm{g}$ de glicose $100 \mathrm{~g}^{-1}$ & \\
\hline \multicolumn{4}{|l|}{ Tratamentos (T) } \\
\hline Água destilada & $55,6 \mathrm{C}$ & $2,0 \mathrm{C}$ & $89,2 \mathrm{C}$ \\
\hline STS $0,2 \mathrm{mM}$ & $53,4 \mathrm{C}$ & $2,0 \mathrm{C}$ & $95,6 \mathrm{~A}$ \\
\hline STS $0,2 \mathrm{mM}+$ sacarose a $50 \mathrm{~g} \mathrm{~L}^{-1}$ & $80,1 \mathrm{~B}$ & $2,8 \mathrm{AB}$ & $90,4 \mathrm{BC}$ \\
\hline STS $0,4 \mathrm{mM}$ & 49,5 D & $2,3 \mathrm{C}$ & $91,9 \mathrm{~B}$ \\
\hline STS $0,4 \mathrm{mM}+$ sacarose a $50 \mathrm{~g} \mathrm{~L}^{-1}$ & $83,6 \mathrm{~A}$ & $3,3 \mathrm{~A}$ & 90,9 B \\
\hline DMS & 2,22 & 0,52 & 1,74 \\
\hline \multicolumn{4}{|l|}{ Dias de vida no vaso $(\mathrm{D})$} \\
\hline 0 & $34,6 \mathrm{C}$ & $2,9 \mathrm{~A}$ & $96,1 \mathrm{~A}$ \\
\hline 3 & $62,8 \mathrm{~B}$ & $2,4 \mathrm{~B}$ & $89,8 \mathrm{~B}$ \\
\hline 6 & $95,9 \mathrm{~A}$ & $2,08 \mathrm{~B}$ & $89,0 \mathrm{~B}$ \\
\hline DMS & 1,43 & 0,34 & 1,15 \\
\hline Interação TxD & ** & ** & ** \\
\hline $\mathrm{CV}(\%)$ & 2,52 & 15,23 & 1,40 \\
\hline
\end{tabular}

Médias seguidas de pelo menos uma letra em comum, para cada variável, não diferem entre si pelo teste de Tukey, ao nível de 5\%, n.s. **, não significativo ou significativo a $\mathrm{P}=0,01$. DMS: diferença mínima significativa; C.V.: coeficiente de variação.

Finger et al. (2004) constataram que, para ave-doparaíso (Strelitzia reginae Banks ex Aiton), o condicionamento das hastes com solução de $1 \mathrm{mM}$ de STS por 30 minutos elevou a longevidade das flores, porém com a adição de $5 \%$ de sacarose a mesma solução reduziu a longevidade dessas. Portanto, não houve efeito aditivo do STS e da sacarose, aplicados na forma de solução de condicionamento, sobre a vida de vaso e sobre a qualidade das inflorescências. Esse mesmo efeito pode ser notado no presente estudo, em que as soluções com STS sem sacarose obtiveram melhores notas de turgescência e cor dos crisântemos (Tabelas 2 e 3).

Tabela 2 - Turgescência das lígulas de crisântemos submetidas a cinco soluções de manutenção e armazenadas por 12 dias, a $22^{\circ} \mathrm{C}$.

\begin{tabular}{lccc}
\hline \multirow{2}{*}{ Soluções de Manutenção } & \multicolumn{3}{c}{ Dias de vida de } \\
& \multicolumn{3}{c}{ vaso } \\
\hline Água Destilada & 4 & 2 & 6 \\
STS 0,2 mM & 4 & 3 & 2 \\
STS 0,2 mM + sacarose a 50 g L & 4 & 2 & 1 \\
STS 0,4 mM & 4 & 3 & 2 \\
STS 0,4 mM + sacarose a 50 g L & 4 & 2 & 1 \\
\hline
\end{tabular}

Critérios: 4 = flores e folhas túrgidas; 3 = levemente murchas; 2 $=$ murchas; $1=$ muito murchas.
Para o parâmetro de cor com atribuição de notas, os tratamentos sem sacarose receberam conceitos melhores ao final das avaliações. Essas notas estão associadas à melhor manutenção da turgescência e menor ocorrência de lesões e murchamento dessas hastes.

Tabela 3 - Cor das lígulas de crisântemos submetidas a cinco soluções de manutenção armazenadas por 12 dias, a $22^{\circ} \mathrm{C}$.

\begin{tabular}{lccc}
\hline \multirow{2}{*}{ Soluções de Manutenção } & \multicolumn{3}{c}{ Dias de vida de } \\
\cline { 2 - 4 } & 0 & 3 & 6 \\
\hline Água Destilada & 4 & 2 & 1 \\
STS 0,2 mM & 4 & 3 & 2 \\
STS 0,2 mM + sacarose a 50 g L & 4 & 3 & 1 \\
STS $0,4 \mathrm{mM}$ & 4 & 3 & 2 \\
STS $0,4 \mathrm{mM}+$ sacarose a $50 \mathrm{~g} \mathrm{~L}^{-1}$ & 4 & 2 & 1 \\
\hline
\end{tabular}

Notas com escala de 1 a 4 , onde 4 = cor viva; $3=$ levemente desbotada; 2 = desbotada; 1 = muito desbotada.

Os tratamentos avaliados não apresentaram contribuição significativa para a abertura de flores (Figura 2). Não houve expansão na largura da lígula e tal resultado pode ser devido à deficiência de água e solutos para a manutenção dos processos metabólicos, independente da associação ou não de sacarose. Para Hassan \& Schmidt (2005), a utilização do STS + sacarose contribuiu para a 
abertura e longevidade floral, exceto para STS 0,4 mM + sacarose que obteve um desempenho semelhante ao controle, apresentando durabilidade de apenas 6 dias.

Na Tabela 4 são apresentados os dados da evolução da luminosidade (onde $0=$ preto e $100=$ branco) em hastes de crisântemos submetidas às soluções de manutenção. Não foi constatada diferença significativa entre as soluções testadas, indicando que essas não influenciaram no escurecimento das flores. Ao longo dos dias de avaliação, verifica-se uma diminuição nos valores de luminosidade, o que significa que as flores tenderam ao escurecimento. Isso pode ser atribuído ao fato das lígulas se apresentarem com déficit hídrico, e apresantam sinais de lesões ao longo dos dias de tratamento.


Figura 2 - Quantidade de botões, flores entreabertas e abertas, durante os 12 dias em que as hastes de crisântemos foram submetidas a cinco soluções de manutenção e armazenadas a $22^{\circ} \mathrm{C}$. 
Tabela 4 - Coloração das lígulas de hastes de crisântemos, submetidas a cinco soluções de manutenção e armazenadas por 12 dias a $22^{\circ} \mathrm{C}$.

\begin{tabular}{lccc}
\hline Variável & Luminosidade & Ângulo de cor & Cromaticidade \\
\hline Tratamentos (T) & & & \\
Água destilada & $32,7 \mathrm{~A}$ & $12,2 \mathrm{~A}$ & $32,7 \mathrm{~A}$ \\
STS 0,2 mM & $32,4 \mathrm{~A}$ & $15,4 \mathrm{~A}$ & $33,3 \mathrm{~A}$ \\
STS 0,2 mM + sacarose a $50 \mathrm{~g} \mathrm{~L}^{-1}$ & $32,8 \mathrm{~A}$ & $9,2 \mathrm{C}$ & $32,5 \mathrm{AB}$ \\
STS 0,4 mM & $32,5 \mathrm{~A}$ & $14,2 \mathrm{~A}$ & $30,7 \mathrm{AB}$ \\
STS 0,4 mM + sacarose a 50 g L & $10,8 \mathrm{BC}$ & 3,24 \\
\hline DMS & $32,6 \mathrm{~A}$ & 2,43 & $36,1 \mathrm{~A}$ \\
\hline Dias de vida no vaso (D) & 2,09 & & $29,1 \mathrm{~B}$ \\
0 & & $18,9 \mathrm{~A}$ & $29,9 \mathrm{~B}$ \\
3 & $33,5 \mathrm{~A}$ & $10,0 \mathrm{~B}$ & 2,13 \\
6 & $32,8 \mathrm{AB}$ & $8,5 \mathrm{~B}$ & $*$ \\
\hline DMS & $31,5 \mathrm{~B}$ & 1,60 & 7,47 \\
\hline Interação TxD & 1,38 & $* *$ & 14,23 \\
\hline CV(\%) & n.s. & 4,69 & \\
\hline
\end{tabular}

Médias seguidas de pelo menos uma letra em comum, para cada variável, não diferem entre si pelo teste de Tukey, ao nível de 5\%, n.s.., **, não significativo ou significativo a $\mathrm{P}=0,01$ e $\mathrm{P}=0,05$, respectivamente. DMS: diferença mínima significativa; C.V.: coeficiente de variação.

O ângulo hue, ou de cor, expressa a manutenção da cor nos produtos avaliados. Com relação a esse atributo foi observado que, em todos os tratamentos, houve tendência de redução nos valores ao longo dos dias (Tabela 4). Essa redução aponta para uma mudança na coloração das lígulas, que passaram de vermelho para vermelho mais profundo, tendendo à púrpura. Dentre os tratamentos, aqueles que apresentaram a melhor manutenção da cor foram os que continham STS 0,2 mM e STS 0,4 mM, sendo que os demais, ao chegarem ao sexto dia, apresentaram desempenho semelhante ao controle que apresentou a maior redução nos valores (Tabela 4). Também na Tabela 4 é possível verificar que os tratamentos diferiram significativamente para a cromaticidade. A cromaticidade variou para os tratamentos avaliados de forma diferente para aqueles que continham a sacarose na sua solução, nos tratamentos que continham o carboidrato houve redução nos valores ao longo do período de avaliação.

\section{CONCLUSÕES}

Nas soluções testadas de STS, a concentração de $0,2 \mathrm{mM}$ foi a que obteve os melhores resultados, prolongando a vida de vaso de crisântemos. A qualidade das hastes foi superior, tendo as melhores avaliações de conteúdo de água, cor e turgescência.

\section{REFERÊNCIAS BIBLIOGRÁFICAS}

ALMEIDA, E. F. A.; PAIVA, P. D. de O.; LIMA, L. C. de O.; RESENDE, M. L.; FONSECA, J.; TAVARES, T. S. Póscolheita de copo-de-leite: Efeito de diferentes conservantes comerciais e armazenamento a frio. Ciência e Agrotecnologia, Lavras, v. 32, n. 4, p. 11891194, jul./ago., 2008.

BARBOSA, J.G.; TAVARES, A.R.R.; FINGER, F.L.; LEITE, R.A. de. Vida de prateleira de minicrisântemos em vaso tratados com tiossulfato de prata. Bragantia, Campinas, v.64, n.4, 2005.

BOROCHOV, A.; MAYAK, S.; BROUN, R. The involvement of water stress and ethylene in senescence of cut carnations flowers. Journal of Experimental Botany, London, v.33, n.137, p.1202-1209, 1982.

BURZO, I.; DOBRESCU, A. The exchange of substances between some cut flowers and solutions during vase life. Acta Horticulturae, Amsterdam, v.405, p.101-107, 1995.

\section{CHANTRACHIT, T. Postharvest physiology of red} ginger inflorescence (Alpinia purpurata (VIEILL) K. Schum.). 1999. 191f. Thesis (Doctor of Philosophy in Horticulturae)-University of Hawaii, Hawaii, 1999. 
FINGER, F.L.; CARNEIRO, T.F.; BARBOSA, J.G. Senescência pós-colheita de inflorescências de esporinha (Consolida ajacis). Pesquisa Agropecuária Brasileira, Brasília, v.39, p.533-537, 2004.

FRANCIS, F.J. Analysis of anthocyanins. In: MARKAKIS, P. (Ed.). Anthocyanins as food colors. New York: Academic, 1982. p.182-205.

HALEVY, A.H. Treatments to improve water balance of cut flowers. Acta Horticulturae, Amsterdam, v.64, p.223230, 1976.

HALEVY, A.H.; MAYAK, S. Improved of cut flower quality opening and longevity by pre-shipment treatments. Acta Horticulturae, Amsterdam, v.43, p.335347, 1974.

HASSAN, F.; SCHMIDT, G. Postharvest characteristics of cut carnations as the result of chemical treatments. Acta Agronomica Hungarica, Akadémiai Kiadó, v.52, n.2, p.125-132, 2005.

HONDA, S.; NISHIMURA, Y.; TAKAHASHI, M.; CHIBA, H.; KAKEHI, K. A manual method for spectrophotometric determination of reducing carbohydrates with 2-cyanoacetaminde. Annals of Chemistry, New York, v.119, p.194-199, 1982.

KRAMER, P.J. Water relations of plants. New York: Academic, 1983. 489p.
LIAO, L.J. et al. Postharvest life of cut rose flowers as affected by silver thiosulphate and sucrose. Botanical Bulletin of Academic Sinica, London, v.41, p.299-303, 2000

MAYAK, S. Senescence of cut flowers. HortScience Amsterdam, v.22, n.5, p.863-868, 1987.

MINOLTA. Precise color communication: color control from feeling to instrumentation. Tokyo, 1994. 49p.

MORAES, P.J.; CECON, P.R.; FINGER, FL.; BARBOSA, J.G.; ALVARES, V.S. Efeito da refrigeração e do condicionamento com sacarose sobre a longevidade de inflorescências de Strelitzia reginae Ait. Revista

Brasileira de Horticultura Ornamental, Campinas, v.5, n.2, p.151-156, 1999.

NOWAK, J.; GOSZCZYNSKA, M.D.; RUDNICKI, R.M. Storage of cut flowers and ornamental plants: present status and future prospects. Postharvest News and Information. Research Institute of Pomology and Floriculture, v.2, n.4, p.255-260, 1991.

PAULIN, A. Influence of exogenous sugar on the evolution of some senescence petals. Acta

Horticulturae, Amsterdam, v.181, p.183-193, June 1986.

VENN, H. Effects of silver salts on the ethylene production and respiration of cut carnations. Acta Horticulturae, Amsterdam, v.91, p.99-103, 1979. 